\title{
Instabilities and doubly spinning black holes
}

\author{
Dumitru Astefanesei, Maria J. Rodriguez*, and Stefan Theisen \\ Max-Planck-Institut für Gravitationsphysik, Albert-Einstein-Institut, 14476 Golm, Germany
}

Received 7 February 2011, accepted 9 February 2011

Published online 1 March 2011

Key words Black holes, thermodynamics, instabilities.

Ultra-spinning black holes in higher dimensions have qualitatively similar thermodynamical properties to the black strings/p-branes- they exhibit a black membrane-like phase. In these notes we will explore which doubly spinning black holes and black rings show this black membrane-like phase and pinpoint the threshold of these regimes. For the same cases we will study the thermodynamical instabilities in the grand canonical ensemble and compute the zeros of the Hessian of the Gibbs potential. Comparing our results, we will show that the thresholds of the black membrane phase and the thermal instability do not necessarily coincide.

() 2011 WILEY-VCH Verlag GmbH \& Co. KGaA, Weinheim

\section{Introduction}

The spectrum of regular stationary asymptotically flat black hole solutions, as solutions of the Einstein equations, is far richer in dimensions bigger than four (see [1] for a concise review). In D space-time dimensions there is more room to allow for solutions with nonspherical event horizon topologies such as $S^{1} \times S^{D-3}$ (black rings [2-4]), $S^{D-2+p} \times T^{p}$ (black p-tuboids [5]), $S^{2} \times S^{D-4}$ (blackfolds [5-7]) and $S^{D-2-p} \times R^{p}$ (black strings/p-branes or in general black membranes [8]).

Interestingly enough, in higher dimensions certain rotating black holes do not show an upper bound on the angular momenta (for fixed mass), and are said to be ultra-spinning [9]. In these regimes the thermodynamical properties of the compact black holes resemble those of the extended black strings/p-branes - or black membranes in general. Moreover, at the level of the metric the geometry of the fast spinning compact black hole resembles that of an extended black hole. This simple observation was then employed to find new approximate analytical black hole solutions through the thin-black ring/blackfold approach by bending the extended black holes $[4,10]$. Identifying which black holes have this membrane-like phases is therefore relevant in this context too. It is our aim to find these transition points to the black membrane-like phase for a wide range of black holes with at least two angular momenta. To extract this information we will conduct a detailed analysis of the thermodynamical properties to clearly define the membrane-like behavior and identify the transitions points.

The existence of the ultra-spinning limit resembling black membranes has a remarkable consequence. Black membranes were shown to be classically unstable [11] so that the ultra-spinning black holes would inherit the Gregory-Laflamme instability. The threshold of the classical instabilities requires a linearized analysis of the perturbations of the black hole solutions that we do not aim to explore here. However as conjectured by [12] (see also [13]) gravitational backgrounds with translationally invariant horizon develop a tachyonic mode (negative non-conformal mode) whenever the specific heat of the black brane geometry becomes negative. By studying the zeros of the Hessian of the Gibbs potential, following [14], we find the threshold of the thermodynamical instabilities of the black holes with at least two angular momenta (in the grand-canonical ensemble) that may correspond to the zero mode of the classical instability.

* Corresponding author E-mail: maria.rodriguez@aei.mpg.de, Phone: +49331 5677154 
One then wonders whether the thresholds of the black membrane phase and the thermal instabilities in the grand canonical ensemble coincide. It was observed in [15] that, for ultra-spinning spherical black holes with only one non trivial angular momentum, there is a tight correspondence between the thresholds of these regimes. A complete thermodynamic analysis, though, should be based on the full Hessian of the thermodynamic potential rather than only a study of the determinant. Comparing our results of the thresholds of the membrane-like phase and the thermodynamical instabilities we find that these two coincide for the spherical black holes with at least two (equal) angular momenta but they do not match for the less symmetric doubly spinning black ring. In this way we find that the connection suggested in [15] is particular to the spherical black holes, but not universal. Further details can also be found in [14].

\section{Framework}

We start by recalling the description of quasilocal formalism [16] supplemented with counterterms. To define the conserved charges we use the divergence-free boundary stress tensor proposed in [17]:

$$
\tau_{i j} \equiv \frac{2}{\sqrt{|h|}} \frac{\delta I}{\delta h^{i j}}=\frac{1}{8 \pi G_{5}}\left(K_{i j}-h_{i j} K-\Psi\left(\mathcal{R}_{i j}-\mathcal{R} h_{i j}\right)-h_{i j} \square \Psi+\Psi_{; i j}\right)
$$

where $\Psi=\sqrt{3} / \sqrt{2 \mathcal{R}}, h_{i j}$ is the induced boundary metric, and $\mathcal{R}_{i j}$ is its Ricci tensor. A rigorous justification and more details about this proposal can be found in [18-20].

Here, $I$ is the renormalized action that includes counterterms,

$$
I=\frac{1}{16 \pi G_{5}} \int_{M} R \sqrt{-g} d^{5} x+\frac{\epsilon}{8 \pi G_{5}} \int_{\partial M}\left(K-\sqrt{\frac{3 \mathcal{R}}{2}}\right) \sqrt{|h|} d^{4} x
$$

$K$ is the extrinsic curvature of $\partial M$ and $\epsilon=+1(-1)$ if $\partial M$ is timelike (spacelike). Moreover, the thermodynamic (effective Gibbs) potential associated to the grand canonical ensemble is

$$
G\left[T, \Omega_{a}\right] \equiv \frac{I}{\beta}=M-T S-\Omega_{a} J_{a}
$$

If the boundary geometry has an isometry generated by Killing vectors $\xi_{t}=\partial_{t}, \xi_{\phi}=\partial_{\phi}$, and $\xi_{\psi}=\partial_{\psi}$, and $\Sigma$ is the hypersurface(with normal $n^{i}$ ) then the conserved charges are

$$
M=\oint_{\Sigma} d^{3} y \sqrt{\sigma} n^{i} \tau_{i j} \xi_{t}^{j}, \quad J_{\phi}=\oint_{\Sigma} d^{3} y \sqrt{\sigma} n^{i} \tau_{i j} \xi_{\phi}^{j}, \quad J_{\psi}=\oint_{\Sigma} d^{3} y \sqrt{\sigma} n^{i} \tau_{i j} \xi_{\psi}^{j} .
$$

\subsection{Doubly spinning black hole}

The Einstein equations in higher dimensions have spinning black hole solutions [21]. We use the explicit black hole metric in Boyer-Lindquist coordinates to compute the boundary stress tensor and we obtain:

$$
\begin{aligned}
\tau_{t t} & =\frac{1}{8 \pi G_{5}}\left(-\frac{3}{2} m \frac{1}{r^{3}}-\frac{5}{3}\left(a^{2}-b^{2}\right) \frac{\cos 2 \theta}{r^{3}}+\mathcal{O}\left(1 / r^{5}\right)\right) \\
\tau_{t \phi} & =\frac{1}{8 \pi G_{5}}\left(-2 a m \frac{\sin ^{2} \theta}{r^{3}}+\mathcal{O}\left(1 / r^{5}\right)\right), \tau_{t \psi}=\frac{1}{8 \pi G_{5}}\left(-2 b m \frac{\cos ^{2} \theta}{r^{3}}+\mathcal{O}\left(1 / r^{5}\right)\right) .
\end{aligned}
$$

Using the definitions (3) and (4) we find the Gibbs potential and the charges respectively. 


\subsection{Doubly spinning black ring}

Some properties of the doubly spinning solution including the structure of the phases in the microcanonical ensemble were discussed in [22]. For a regular black ring solution, the parameters $\nu$ and $\lambda$ are constrained to satisfy $0 \leq \nu<1,2 \sqrt{\nu} \leq \lambda<1+\nu$. In the limit $\nu \rightarrow 0$ the black ring with one angular momentum $\left(J_{\phi}\right)$ is recovered $\left(J_{\psi}\right.$ is the angular momentum on $\left.S^{2}\right)$. The limit $\lambda \rightarrow 2 \sqrt{\nu}$ was carefully studied in [22] and shown to correspond to regular extremal black rings.

The components of the boundary stress tensor relevant to our studies in the coordinates of [22] are

$$
\begin{aligned}
\tau_{t t} & =\frac{1}{8 \pi G_{5}}\left(-\frac{12 k^{2} \lambda}{(1+\nu-\lambda)} \frac{1}{r^{3}}-\frac{8 k^{2} F_{1}[\nu, \lambda]}{3(1+\nu-\lambda)(1-\nu)^{2}} \frac{\cos 2 \theta}{r^{3}}+\mathcal{O}\left(1 / r^{5}\right)\right), \\
\tau_{t \phi} & =\frac{1}{8 \pi G_{5}}\left(\frac{16 k^{3} \lambda\left(1+\lambda-6 \nu+\lambda \nu+\nu^{2}\right) \sqrt{(1+\nu)^{2}-\lambda^{2}}}{(1+\nu-\lambda)^{2}(1-\nu)^{2}} \frac{\sin ^{2} \theta}{r^{3}}+\mathcal{O}\left(1 / r^{5}\right)\right), \\
\tau_{t \psi} & =\frac{1}{8 \pi G_{5}}\left(\frac{32 k^{3} \lambda \sqrt{\nu\left[(1+\nu)^{2}-\lambda^{2}\right]}}{(1+\nu-\lambda)(1-\nu)^{2}} \frac{\cos ^{2} \theta}{r^{3}}+\mathcal{O}\left(1 / r^{5}\right)\right) .
\end{aligned}
$$

where $F_{1}[\nu, \lambda]=1-5 \nu-\nu^{2}+5 \nu^{3}+\lambda^{2}(3+7 \nu)+\lambda\left(1-14 \nu-7 \nu^{2}\right)$. Using (3) we find $G=\pi k^{2} \lambda /(1+\nu-\lambda)$, and by plugging the expressions (6) in (4) we find the expressions for the conserved charges.

\section{Membrane-like phases of black holes}

The transition of black holes [21], from behaving like a spherical black hole to behaving like a black membrane as the spin grows, was established to be at the minimum of the temperature [9]. From that point onwards, the temperature increases in a similar way as for the black brane temperature.

The same result was obtained by using a different method [23], namely finding the divergences of the 'Ruppeiner curvature' [24]. The Ruppeiner curvature, which is the scalar curvature of the Hessian matrix of the entropy, blows-up exactly at the same value signaling a thermal instability of the system.

However, the transition to a membrane-like phase of the rapidly spinning black holes can be established from the study of their thermodynamics. The location of the threshold of this regime is signaled by the minimum of the temperature and the maximum angular velocity as functions of the angular momentum.

It was observed in [15] that, for ultra-spinning black holes, this is in tight correspondence with a vanishing eigenvalue of the Hessian of the Gibbs potential. A complete thermodynamic analysis, though, should be based on the full Hessian of the thermodynamic potential rather than only a study of the determinant. We will see in the next subsection that the membrane phase of a doubly spinning ring is not signaled by a zero-eigenvalue of the Gibbs potential's Hessian.

For ultra-spinning black holes, there is a direct relation between (response functions in) the microcanonical and grand canonical ensembles. To see that, we compare the following response functions:

$$
\left(\frac{\partial^{2} S}{\partial J^{2}}\right)_{M}=-\frac{1}{T}\left(\frac{\partial \Omega}{\partial J}\right)_{M}+\frac{\Omega}{T^{2}}\left(\frac{\partial T}{\partial J}\right)_{M} \quad \text { and } \quad\left(\frac{\partial^{2} G}{\partial \Omega^{2}}\right)_{T}=-\left(\frac{\partial J}{\partial \Omega}\right)_{T}
$$

We have checked that in the particular case of the singly spinning black hole, indeed, these two response functions are inverse proportional at the particular point where the temperature has a minimum. Therefore, an inflexion point in the microcanonical ensemble corresponds to a divergence of the corresponding response function in the grand canonical ensemble. This may well be an explanation for the results obtained in [15]. Moreover, this point should not be considered as a sign for an instability or a new branch but a transition to an infinitesimally nearby solution along the same family of solutions. The numerical evidence of [15] supports this connection with the zero-mode perturbation of the solution. 
We now examine the situation for a more general family of ultra-spinning Myers-Perry black holes with multiple spin parameters, $a_{i}$, where $i=1,2, \ldots, N$ and $N=[(D-1) / 2]$. The black hole is characterized by the mass parameter $\mu$ and the horizon radius $r_{h}$ by which we can express the thermodynamics.

A sufficient, but not necessary, condition for the existence of ultra-spinning black holes was given in [9]. In even(odd) dimensions at least one(two) of the spins should be much smaller than the rest. The ultra-spinning regime is obtained in the limit

$$
0 \leq a_{1}, a_{2}, \ldots, a_{k} \ll a_{k+1}, \ldots, a_{N} \rightarrow \infty
$$

where $N-1 \geq k \geq 1+\epsilon$ where $\epsilon=+1$ for odd- $D$ and $\epsilon=0$ otherwise. Either static, with all finite angular momenta $a_{1}, \ldots, a_{k}$ vanishing, or spinning, with some $a_{1}, \ldots, a_{k}$ non-vanishing, the generic limiting black brane is $S^{D-2(N-k+1)} \times \mathbb{R}^{2(N-k)}$.

Our focus will be on the case in which the black hole has at least two large spins and we set the remaining angular momenta to zero. When the angular momenta are equal, $J_{k+1}=\ldots=J_{N}=J$, the Ruppeiner curvature scalar blows up at

$$
\frac{a^{2}}{r_{h}^{2}}=\frac{D-3}{2 k-1-\epsilon} .
$$

When $k=k_{\max }$ our results agree with those of [23]. and according to [24], this signals a thermodynamic instability. However, the expected new phase should correspond to the black membrane phase of ultraspinning black holes and not to a new branch of solutions. Indeed, we find that the divergences of the Ruppeiner curvature pinpoint the zero of the determinant of the Gibbs potential's Hessian.

Also, by studying the temperature

$$
T=\frac{(D-3)\left(1+\frac{n}{(D-3)} \frac{4 J^{2}}{S^{2}}\right)}{4 S^{\frac{1}{D-2}}\left(1+\frac{4 J^{2}}{S^{2}}\right)^{\frac{D-1+n}{2(D-2)}}}, \quad n=2 k-1-\epsilon
$$

we find that the temperature has a minimum at exactly (9), while the angular velocity $\Omega$ reaches its maximum value. Therefore for these more general ultra-spinning black holes, similarly to the singly spinning situation discussed in [9], once the minimum is reached the temperature increases and the angular velocity decreases signaling a transition to a membrane phase. This conduct is shown in Fig. 2 (points $I$ on the solid thin line) for the particular case of $D=7, k=2$ so $j_{1}=j_{2} \equiv j_{\phi}$ and $j_{3}=0$.

\section{Thermodynamic instabilities of black rings}

For a black ring with one angular momentum the heat capacity can be positive definite, but the momentum of inertia is always negative [26]. Therefore, the singly spinning black ring is thermodynamically unstable. As in the case of one angular momentum, the heat capacity of a doubly spinning black ring can be positive in some region of the parameter space. However, there is a key difference when the second angular momentum is turned on. That is, the component of the momentum of inertia associated to $S^{1}$ of the black ring can become positive - this is explicitly shown in Fig. 1.

Since there are two angular momenta one should also investigate the effect of coupled 'angular' inhomogeneities. A careful study of the determinant of the momentum of inertia matrix shows that there is no region in the parameter space with the desired properties and so the doubly spinning black ring is also thermodynamically unstable.

\section{Membrane-like phase of black rings}

For the singly spinning black ring, an analysis of the temperature as a function of the angular momentum was presented in [25]. In this case (solid thick line in the plot on the left hand side of Fig. 2), the temperature 


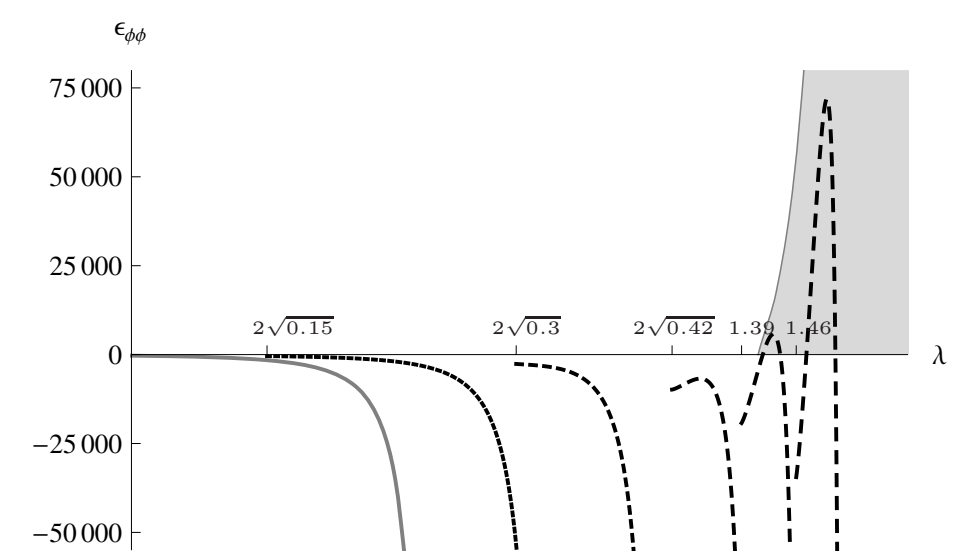

Fig. 1 Plot of the response function $\epsilon_{\phi \phi}$ as a function of $\lambda$. The gray curve corresponds to the compressibility of the singly spinning black ring, namely $\nu=0$. As the angular momentum along the $S^{2}$ is increased (dashed line left towards right) the isothermal moment of inertia for different values of $\nu=0.15,0.3,0.42,0.48,0.53$ changes and becomes positive for values of $\nu>0.46690042$ and $\lambda>1.40685$ (shaded gray region).

does not have a minimum, but there exists a turning point. In our analysis, the turning point $I I$ for the black ring plays a similar role as the minimum of the temperature for the black hole. That is, it signals a change in the thermodynamic behavior of the black ring. In fact, it is the starting point of the ultra-spinning regime where the black ring can be approximated by a boosted black string.

Using the Poincaré 'turning point' method, this special point was carefully studied in [25]. In particular, they found a divergence of the Ruppeiner curvature. In the conjugacy diagram (on the right) there is also a turning point $I I$ at the same minimum value of the angular momentum $j_{\phi}$.

The question is then if there still is a relation between the microcanonical and grand canonical ensembles in this case. We have explicitly checked, using the results of [26], that one of the eigenvalues of the Hessian of the Gibbs potential is zero at this specific point $I I$ while the second eigenvalue never changes its sign. Therefore, we conclude that the turning point is the onset of the ultra-spinning black string phase.

A far more richer structure is found for the doubly spinning black ring. The angular momentum on $S^{2}$ is bounded as $j_{\psi} \in[0,1 / 4]$ and for a specific $j_{\psi}$ the black ring can always be extremal (in the limit $\lambda \rightarrow 2 \sqrt{\nu}$ as shown in [22]). But besides extremality, according to how large $j_{\psi}$ is, the behavior of the doubly spinning black ring changes. There are two distinctive regions in the microcanonical ensemble. On one hand, for $0 \leq j_{\psi}<1 / 5$, there are phases with the characteristic cusp for black rings with the two (fat and thin) branches. But, for $1 / 5 \leq j_{\psi} \leq 1 / 4$, there are no cusps, hence no fat black ring branch. This will become relevant to understand the physics and regimes of the doubly spinning black ring.

To explore how the physics of the black rings at fixed mass is modified as we turn on the angular momenta along the $S^{2}, j_{\psi}$, we will study the temperature and angular velocity as functions of the $S^{1}$ angular momentum, $j_{\phi}$, for different fixed values of $j_{\psi}$.

Doubly spinning black rings with $0 \leq j_{\psi}<\mathbf{1 / 5}$. In this case the situation is similar, to some extent, to what we found before for the singly spinning black ring. In this range, as we turn on $j_{\psi}$, there also are turning points with tangents of infinite slope signaling the onset of the black membrane phase that coincide with the cusps (in parameter space), namely at $\lambda=-(1 / 4)\left(1+\nu-(9+\nu)^{1 / 2}(1+9 \nu)^{1 / 2}\right)$. Figure 2 shows this change in behavior explicitly. On the left (light gray curve) in the $\mathrm{t} v \mathrm{v} . j_{\phi}$ diagram, we observe the turning point $I I I$ that corresponds to the minimum value of $j_{\phi}$ angular momentum. In the conjugacy diagram $\omega$ vs. $j_{\phi}$ (also the light gray curve), the corresponding point $(I I I)$ is a turning point. 

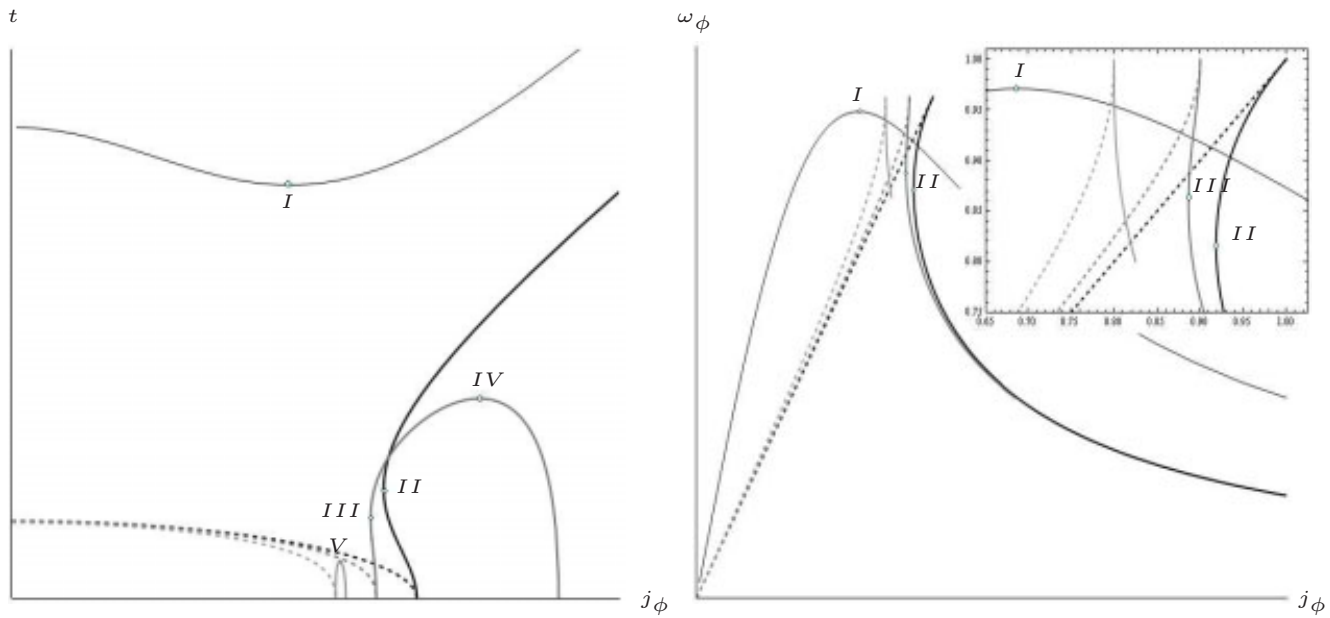

Fig. 2 Plots of the temperature (left hand side) and angular velocity (right hand side) as functions of the angular momentum $j_{\phi}$, for a fixed mass, for different black objects (we employ the dimensionless quatities as in [4]). These include the singly spinning Myers-Perry black holes in five dimensions of space-time (black dashed line) and its seven dimensional cousin (solid thin line). The singly (solid thick) and doubly spinning black ring (light gray) for different values of angular momenta (right towards left) $j_{\psi}=0.1,0.2$ on the $S^{2}$, are also shown here. For this same values of the second angular momenta the five-dimensional doubly spinning black holes are represented by the dashed light gray lines.

But an interesting difference with the single spin black ring occurs for a large enough $S^{1}$ angular momentum. The temperature of the black ring in its membrane phase increases while the area of the event horizon decreases up to a point where the spin-spin interaction is large enough making a turn to abruptly become extremal with zero temperature. This is the maximum critical value labeled $I V$ in Fig. 2 . Therefore, the black membrane phase exists between points $I I I$ and $I V$. Finally, we computed the eigenvalues of the $\operatorname{Hess}[G]$ and found that none of them are zero the relevant turning points.

Doubly spinning black rings with $1 / 5 \leq j_{\psi} \leq 1 / 4$. This black rings (lightest gray line in Fig. 2) with larger $S^{2}$ angular momentum show no turning points and therefore have no membrane phase. This mimics its behavior in the phase diagram of microcanonical ensemble where the cusp and fat branch of these doubly spinning black rings disappear [22]. The lack of a fat black ring branch seems to coincide with the lack of a black membrane phase. Therefore such solutions would never be captured with long distance effective approaches $[4,10]$.

Note that for certain fixed $j_{\psi} \in[1 / 5,1 / 4]$ the temperature grows, reaching a maximum at $V$ and rapidly decreasing to zero to become extremal. It would be interesting to explore the physical meaning of these points which we observe to correspond to an inflection point $\partial^{2} S / \partial^{2} J=0$ for fixed mass.

\section{References}

[1] M. J. Rodriguez, Twelfth Marcel Grossmann Meeting on General Relativity [arXiv:1003.2411 [hep-th]].

[2] R. Emparan and H. S. Reall, Phys. Rev. Lett. 88, 101101 (2002) [arXiv:hep-th/0110260].

[3] A. A. Pomeransky and R. A. Sen'kov, arXiv:hep-th/0612005.

[4] R. Emparan, T. Harmark, V. Niarchos, N. A. Obers, and M. J. Rodriguez, J. High Energy Phys. 0710, 110 (2007) [arXiv:0708.2181 [hep-th]].

[5] R. Emparan, T. Harmark, V. Niarchos, and N. A. Obers, J. High Energy Phys. 1004, 046 (2010) [arXiv:0912.2352 [hep-th]]. 
[6] B. Kleihaus, J. Kunz, and E. Radu, Phys. Lett. B 678, 301 (2009) [arXiv:0904.2723 [hep-th]].

[7] B. Kleihaus, J. Kunz, E. Radum, and M. J. Rodriguez, Accepted for publication in J. High Energy Phys. [arXiv: 1010.2898 [gr-qc]].

[8] G. T. Horowitz and A. Strominger, Nucl. Phys. B 360, 197 (1991).

[9] R. Emparan and R. C. Myers, J. High Energy Phys. 0309, 025 (2003) [arXiv:hep-th/0308056].

[10] R. Emparan, T. Harmark, V. Niarchos, and N.A. Obers, J. High Energy Phys. 1003, 063 (2010) [arXiv:0910.1601 [hep-th]].

[11] R. Gregory and R. Laflamme, Phys. Rev. Lett. 70, 2837 (1993) [arXiv:hep-th/9301052].

[12] S. S. Gubser and I. Mitra, J. High Energy Phys. 0108, 018 (2001) [arXiv:hep-th/0011127].

[13] H. S. Reall, Phys. Rev. D 64, 044005 (2001), [arXiv:hep-th/0104071].

[14] D. Astefanesei, M. J. Rodriguez, and S. Theisen, J. High Energy Phys. 1008, 046 (2010) [arXiv:1003.2421 [hep-th]].

[15] O. J.C. Dias, P. Figueras, R. Monteiro, J.E. Santos, and R. Emparan, Phys. Rev. D 80, 111701 (2009) [arXiv:0907.2248 [hep-th]].

[16] J. D. Brown and J. W. . York, Phys. Rev. D 47, 1407 (1993) [arXiv:gr-qc/9209012].

[17] D. Astefanesei and E. Radu, Phys. Rev. D 73, 044014 (2006) [arXiv:hep-th/0509144].

[18] R. B. Mann and D. Marolf, Class. Quantum Gravity 23, 2927 (2006) [arXiv:hep-th/0511096].

[19] R. B. Mann, D. Marolf, and A. Virmani, Class. Quantum Gravity 23, 6357 (2006) [arXiv:gr-qc/0607041].

[20] D. Astefanesei, R. B. Mann, and C. Stelea, Phys. Rev. D 75, 024007 (2007) [arXiv:hep-th/0608037].

[21] R. C. Myers and M. J. Perry, Ann. Phys. (Berlin) 172, 304 (1986).

[22] H. Elvang and M. J. Rodriguez, J. High Energy Phys. 0804, 045 (2008) [arXiv:0712.2425 [hep-th]].

[23] J.E. Aman and N. Pidokrajt, Phys. Rev. D 73, 024017 (2006) [arXiv:hep-th/0510139].

[24] G. Ruppeiner, Rev. Mod. Phys. 67, 605 (1995); Erratum, Rev. Mod. Phys. 68, 313 (1996).

[25] G. Arcioni and E. Lozano-Tellechea, Phys. Rev. D 72, 104021 (2005) [arXiv:hep-th/0412118].

[26] D. Astefanesei, R. B. Mann, M. J. Rodriguez and C. Stelea, Class. Quantum Gravity 27, 165004 (2010) [arXiv:0909.3852 [hep-th]]. 